Invited Paper

\title{
Engineering complex nanolasers: from spaser quantum information sources to near-field lasers
}

\author{
Juan Sebastian Totero Gongora ${ }^{\mathrm{a}}$, Andrey E. Miroshnichenko ${ }^{\mathrm{b}}$, Yuri S. Kivshar ${ }^{\mathrm{b}}$, and Andrea \\ Fratalocchi $^{\mathrm{a}}$ \\ aPRIMALIGHT, King Abdullah University of Science and Technology (KAUST), Thuwal \\ 23955-6900, Saudi Arabia \\ ${ }^{b}$ Nonlinear Physics Centre, Australian National University, Canberra ACT 2601, Australia.
}

\begin{abstract}
In this invited contribution I will review recent results of our research in the field of complex nanolasers. I will begin by discussing recent experimental results from a new type of ultra-dark nanoparticles, which behave as an ideal black-body and spontaneously produce single color pulses thanks to an equivalent Bose-Einstein Condensation of light. ${ }^{1}$ I will then discuss new quantum information sources from core-shell spaser nanoparticles. ${ }^{2}$ Finally, I will illustrate a new type of laser source that emits only in the near field, discussing applications in integrated optical circuits.
\end{abstract}

Keywords: spaser, anapole, rotating dipole, nonlinear dynamics, integrated nanolasers

\section{INTRODUCTION}

In recent years, researchers have devoted significant efforts in the development of integrated sources at the nanoscale. ${ }^{3-5}$ While significant results have been achieved by combining semiconductor devices and silicon photonics, further advances in this field require to address many fundamental challenges, originating from the nanoscale miniaturization of optical components. One of the major challenges is the diffraction limit of light, which introduces significant radiative losses that are detrimental and severely limit the performance of nanoscale devices. ${ }^{6,7}$ In order to circumvent these issues, several devices and configurations have been proposed, aiming at the localization and amplification of the electromagnetic field in sub-wavelength structures. Among such possible candidates, the SPASER represents one of the most interesting solutions. In a spaser device a plasmonic resonance of the structure is amplified by the presence of a resonant medium. ${ }^{8}$ When the incident pumping power exceeds the spasing threshold of the system, the spaser can sustain fully optical and coherent emission, which has been postulated theoretically and observed experimentally. ${ }^{9-11}$ Despite their peculiar properties, however, spaser-based nanolasers are affected by extremely large losses, due to the interaction between the electromagnetic fields and the metallic nanoparticles. Therefore, significant efforts have been devoted to the development of alternative integrated nanolasers based on all-dielectric configurations. Recently, intriguing non-radiating electromagnetic modes, known as anapoles, have been experimentally discovered in silicon nanoparticles. ${ }^{12}$ Anapole states are generated by the superposition of electric and toroidal dipole modes, which cancel each other in the far-field. As a result, the scattered field from an anapole state is entirely localized in the near-field. ${ }^{13,14}$ Moreover, in contrast to plasmonic resonances, anapole modes can provide an extremely high degree of localization without introducing radiative and dissipative losses. Due to their unique properties, therefore, anapole states represent an ideal candidate for the development of a new type of all-dielectric near-field nanolasers. ${ }^{15}$

In this work, we discuss the emission dynamics from core-shell spasers and anapole-based nanolasers by means of three-dimensional Finite-Differences Time-Domain (FDTD) ab-initio simulations. ${ }^{16}$ Due to their non-conventional properties, the detailed analysis of the dynamics originated in these nanolasing systems represents a challenging task. In fact, standard classical and semi-classical methods usually provide limited information on the temporal dynamics of the spontaneous/stimulated transition and on the evolution and mutual interaction of the

Further author information: (Send correspondence to A.F.)

A.F.: E-mail: andrea.fratalocchi@kaust.edu.sa

Integrated Optics: Devices, Materials, and Technologies XXI, edited by Sonia M. García-Blanco, Gualtiero Nunzi Conti, Proc. of SPIE Vol. 10106, 101061B · @ 2017 SPIE

CCC code: $0277-786 \mathrm{X} / 17 / \$ 18 \cdot$ doi: $10.1117 / 12.2249497$

Proc. of SPIE Vol. 10106 101061B-1 
spatial modes of the system before and during the lasing stage. The description of these dynamics requires the development of a rigorous time dependent method in which the amplifying medium is described in a fully quantum-mechanical framework. In order to address some of these challenges, we implemented a set of vectorial Maxwell-Bloch (MB) equations into the FDTD algorithm, unveiling an extremely rich physical scenario underlying the femtosecond emission dynamics of these nanolaser systems.

\section{MAXWELL-BLOCH FDTD APPROACH}

The rigorous ab-initio simulation of an integrated nanolaser requires a fully quantum-mechanical description of the amplifying medium and the explicit inclusion of material dispersion (e.g., in the case of plasmonic structures). The total response of the system is given by a total polarization field $\mathbf{P}_{T}$, which can be divided into its linear and nonlinear contributions as $\mathbf{P}=\mathbf{P}_{\text {lin }}+\mathbf{P}_{\text {Nlin }}$. The linear polarization $\mathbf{P}_{\text {lin }}$ takes into account all the linear dispersion effects, i.e. $\mathbf{P}_{l i n}(t)=\varepsilon_{0} \int \mathrm{d} t^{\prime} \chi_{l i n}\left(t-t^{\prime}\right) \mathbf{E}\left(t^{\prime}\right)$. The nonlinear polarization $\mathbf{P}_{n l i n}$, conversely, includes the light-matter interaction phenomena occurring in the amplifying medium. The evolution of the system follows Maxwell's equations:

$$
\left\{\begin{array}{l}
\frac{\partial \mathbf{H}}{\partial t}=-\frac{1}{\mu_{0}} \nabla \times \mathbf{E} \\
\frac{\partial \mathbf{E}}{\partial t}=\frac{1}{\varepsilon_{0}}\left[\nabla \times \mathbf{H}-\frac{\partial}{\partial t}\left(\mathbf{P}_{l i n}+\mathbf{P}_{n l i n}\right)\right]
\end{array}\right.
$$

with opportune additional equations for the polarization terms. ${ }^{16,17}$ In our numerical approach, we represent the resonant medium by means of a four-level atomic system, composed of a ground state and three excited states, which account for independent transitions. The energy difference between the excited and ground states is set as $\hbar \omega_{0}$ and the evolution of the quantum-mechanical system is described using the density operator formalism. The evolution of the nonlinear polarization is dictated by the Maxwell-Bloch (MB) equations, which read

$$
\left\{\begin{array}{l}
\mathbf{P}_{N l i n}=e q_{0} N_{a}\left[S_{1} \hat{\mathbf{x}}+S_{4} \hat{\mathbf{y}}+S_{9} \hat{\mathbf{z}}\right] \\
\partial_{t} S_{l}=\sum \Gamma_{l m} S_{m}-\gamma_{l l}\left[S_{l}-S_{l}^{(0)}\right] \\
\Gamma_{l m}=\frac{j}{2 \hbar} \operatorname{Tr}\left\{H\left[\lambda_{l}, \lambda_{m}\right]\right\}, \gamma_{i j}=1 / \tau_{i j} .
\end{array}\right.
$$

Here $S_{j}$ is the j-th component of the Bloch coherence vector, $N_{a}$ is the density of polarizable atoms in the medium, $\lambda_{i}$ is the $\mathrm{i}$-th generator of the $\mathrm{SU}(4)$ group, $\tau_{i j}$ are decay times and $H$ is the Hamiltonian of the atomic system. ${ }^{18}$ A full derivation of Eqs. (2) and the description of all the physical quantities can be found in. ${ }^{19,20}$ We solved Eqs. (1) and (2) inside the Finite-Differences-in-Time-Domain (FDTD) framework using our massively parallel FDTD simulator NANOCPP. ${ }^{21-23}$ More specifically, we used a novel computational approach which explicitly takes into account the presence of material dispersion. In all our simulations the computational domain was organized as follows: the nanolaser was placed in the center of a box with $2 \mu \mathrm{m}$ side, while the spatial resolution in air was set as $2.5 \mathrm{~nm}$. In order to simulate an open system, we used Uniaxial Perfectly Matched Layer (UPML) boundary conditions. ${ }^{16}$ Additionally, in our simulations we explicity considered the presence of quantum noise, whose amplitude is set in terms of the system temperature $T .^{24}$

\section{ROTATIONAL EMISSION FROM AN INTEGRATED SPASER}

As a first example, we investigated the emission from a single core-shell spaser, composed by an Ag core embedded in a Rh800-doped silica shell. Such configuration corresponds to a typical experimental geometry, opening to a direct comparison with published experimental results. ${ }^{10}$ In order to characterize the spasing action, we conducted an extensive campaign of simulations for a three-dimensional core-shell structure and for different values of the pumping power $N_{a} .{ }^{2}$ Interestingly, above the spasing threshold the emitted field is characterized by different spatial phases, including a surprising rotational emission illustrated in Fig. 1-a,c Such purely rotating emission pattern occurs for values of $N_{a}>2.5 \mathrm{part} / \mathrm{m}^{3}$ and it is characterized by an angular rotation equal to the emission frequency $\omega_{0}$ (Fig. 1-f), $N_{a}=3 \times 10^{27}$ ). Even more interestingly, due to to the presence of 


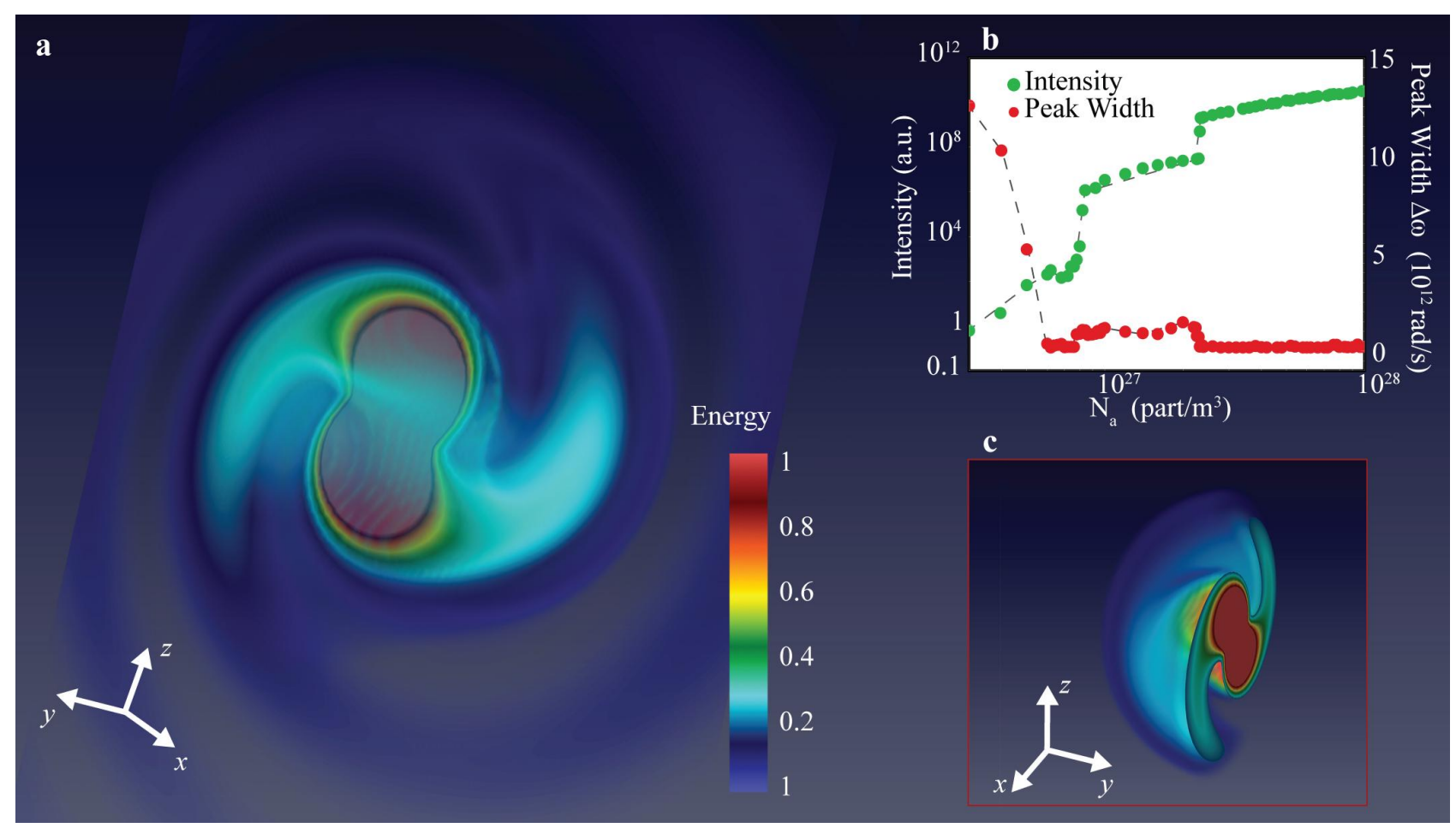

Figure 1. FDTD analysis of a three-dimensional spaser. a,c Volumetric plot of the electromagnetic energy in a three-dimensional spaser system for $N_{a}=2 \times 10^{27}$. The rotational plane is selected by means of the incident polarization. b Input-output diagram of the spaser emission as a function of the input power, describing the characteristic "S"-shaped curve in the LL power plot (green dots) and the spectral narrowing (red dots).

quantum noise, the direction of rotation of the emitted field changes randomly across different realizations of the spaser. As discussed in ${ }^{2}$ the occurrence of a purely rotational emission is the result of a complex form of modal competition among an infinite number of spatially degenerate modes. In the presence of strong non-linear interactions, in fact, the emission from the system is dominated by energy equipartition, which redistributes the electromagnetic energy along all the angular directions and generates the purely rotational evolution observed in our simulations. The occurrence of a true spasing transition is confirmed by the spectral narrowing and the LL plot of the emitted field, which are shown in Fig. 1-b. The characteristic signature of a spasing transition is the "S"-shaped evolution of the intensity peak versus density $N_{a}$ (green dots), as well as by the exponential narrowing of the emission linewidth (red dots), which approaches an almost constant value in the stationary spasing regime. Interestingly, in the LL-plot the transition from a single-mode spasing to the fully rotational emission is marked by the occurrence of a seond high-power transition. The final linewidth of the spaser is of the order of $1-2 \mathrm{~nm}$, which is in quantitative agreement with experimental results. ${ }^{25,26}$ The remarkable similitude of Fig. 1-b with Fig. 3-b of, ${ }^{25}$ suggests the possibility that the spasing rotational dynamics can be observed within the pumping ranges of current experiments.

\section{NONLINEAR AMPLIFICATION OF RADIATION-LESS MODES}

In order to simulate a realistic anapole-based nanolaser, we considered a monolithic III-V semiconductor cylindrical resonator. As an incident polarization, we consider an electric field perpendicular to the cylinder axis. Interestingly, the dielectric nanolaser, emitting at the anapole frequency, achieves a stationary lasing emission from a InGaAs anapole laser with radius $r \approx 450 \mathrm{~nm}$ and height $h=100 \mathrm{~nm}$. Due to frequency pulling effects, the emission frequency is slightly shifted with respect to the linear anapole frequency. Interestingly, the emitted energy is entirely confined in the near-field. This opens many new interesting applications for compact and integrated sources of light. 


\section{CONCLUSIONS}

By developing a rigorous time-dependent ab-initio model, we investigated the ultrafast dynamics of two peculiar types of three-dimensional integrated nanolasers. By considering a core-shell spaser nanolaser, we have shown how the core-shell nanoparticle, despite its simple geometry, exhibits a remarkable complexity that is able to sustain a rich scenario of different phases, including a fully stable rotational emission. ${ }^{2}$ By coupling the rotational emission with external optical components, it can be shown how our results open to the attractive perspective of using spasers as a new type of unidirectional source. ${ }^{27,28}$

As an additional case study, we also investigated the emission dynamics from an all-dielectric nanolaser emitting at the anapole mode. Our results show how, despite its peculiar properties, anapole states are able to sustain stimulated emission of radiation. Interestingly, the lasing emission from anapole-based nanolasers is entirely confined in the near-field.

\section{Acknowledgment}

For the computer time, we have used the resources of the KAUST Supercomputing Laboratory. This work is part of the research program of Kaust "Optics and plasmonics for efficient energy harvesting" (Award No. CRG-1-2012-FRA-005) and it was also supported by the Australian Research Council.

\section{REFERENCES}

[1] Huang, J., Liu, C., Zhu, Y., Masala, S., Alarousu, E., Han, Y., and Fratalocchi, A., "Harnessing structural darkness in the visible and infrared wavelengths for a new source of light," Nature Nanotechnology 11, 60-66 (Jan. 2016).

[2] Totero Gongora, J. S., Miroshnichenko, A. E., Kivshar, Y. S., and Fratalocchi, A., "Energy equipartition and unidirectional emission in a spaser nanolaser," Laser and Photonics Reviews 10(3), 432-440 (2016).

[3] Rupasinghe, C., Rukhlenko, I. D., and Premaratne, M., "Spaser made of graphene and carbon nanotubes," ACS Nano 8(3), 2431-2438 (2014).

[4] Ma, R.-M., Ota, S., Li, Y., Yang, S., and Zhang, X., "Explosives detection in a lasing plasmon nanocavity," Nat. Nanotechnol. 9, 600-604 (08 2014).

[5] Sidiropoulos, T. P. H., Roder, R., Geburt, S., Hess, O., Maier, S. A., Ronning, C., and Oulton, R. F., "Ultrafast plasmonic nanowire lasers near the surface plasmon frequency," Nat. Phys. 10, 870-876 (09 2014).

[6] Kryzhanovskaya, N. V., Zhukov, A. E., Maximov, M. V., Moiseev, E. I., Shostak, I. I., Nadtochiy, A. M., Kudashova, Y. V., Lipovskii, A. A., Kulagina, M. M., and Troshkov, S. I., "Room Temperature Lasing in 1\#x03bc;m Microdisk Quantum Dot Lasers," IEEE Journal of Selected Topics in Quantum Electronics 21, 709-713 (Nov. 2015).

[7] Khurgin, J. B. and Sun, G., "Comparative analysis of spasers, vertical-cavity surface-emitting lasers and surface-plasmon-emitting diodes," Nature Photonics 8, 468-473 (June 2014).

[8] Bergman, D. J. and Stockman, M. I., "Surface plasmon amplification by stimulated emission of radiation: Quantum generation of coherent surface plasmons in nanosystems," Phys. Rev. Lett. 90, 027402-027405 (Jan 2003).

[9] Zheludev, N. I., Prosvirnin, S. L., Papasimakis, N., and Fedotov, V. A., "Lasing spaser," Nat. Photon. 2, 351-354 (06 2008).

[10] Noginov, M. A., Zhu, G., Belgrave, A. M., Bakker, R., Shalaev, V. M., Narimanov, E. E., Stout, S., Herz, E., Suteewong, T., and Wiesner, U., "Demonstration of a spaser-based nanolaser," Nature 460, 1110-1112 (08 2009).

[11] Zhang, Q., Li, G., Liu, X., Qian, F., Li, Y., Sum, T. C., Lieber, C. M., and Xiong, Q., "A room temperature low-threshold ultraviolet plasmonic nanolaser," Nat. Commun. 5 (09 2014).

[12] Miroshnichenko, A. E., Evlyukhin, A. B., Yu, Y. F., Bakker, R. M., Chipouline, A., Kuznetsov, A. I., Lukyanchuk, B., Chichkov, B. N., and Kivshar, Y. S., "Nonradiating anapole modes in dielectric nanoparticles," Nature Communications 6, 8069 (Aug. 2015). 
[13] Liu, W., Zhang, J., Lei, B., Hu, H., and Miroshnichenko, A. E., "Invisible nanowires with interfering electric and toroidal dipoles," Optics Letters 40, 2293 (May 2015).

[14] Fan, P., Chettiar, U. K., Cao, L., Afshinmanesh, F., Engheta, N., and Brongersma, M. L., "An invisible metal-semiconductor photodetector," Nature Photonics 6, 380-385 (June 2012).

[15] Huang, Y.-W., Chen, W. T., Wu, P. C., Fedotov, V. A., Zheludev, N. I., and Tsai, D. P., "Toroidal Lasing Spaser," Scientific Reports 3, 1237 (Feb. 2013).

[16] Taflove, A. and Hagness, S. C., [Computational electrodynamics : the finite-difference time-domain method], Artech House antennas and propagation library, Artech House, Boston, 3rd ed. (2005).

[17] Taflove, A., Oskooi, A., and Johnson, S. G., [Advances in FDTD computational electrodynamics : photonics and nanotechnology], Artech House antennas and propagation series, Artech House (2013).

[18] Ziolkowski, R. W., Arnold, J. M., and Gogny, D. M., "Ultrafast pulse interactions with two-level atoms," Phys. Rev. A 52(4), 3082-3094 (1995).

[19] Fratalocchi, A., Conti, C., and Ruocco, G., "Three-dimensional ab initio investigation of light-matter interaction in mie lasers," Physical Review A 78(1) (2008).

[20] Conti, C. and Fratalocchi, A., "Dynamic light diffusion, three-dimensional anderson localization and lasing in inverted opals," Nat. Phys. 4, 794-798 (10 2008).

[21] Coluccio, M. L., Gentile, F., Das, G., Nicastri, A., Perri, A. M., Candeloro, P., Perozziello, G., Proietti Zaccaria, R., Gongora, J. S. T., Alrasheed, S., Fratalocchi, A., Limongi, T., Cuda, G., and Di Fabrizio, E., "Detection of single amino acid mutation in human breast cancer by disordered plasmonic self-similar chain," Science Advances 1(8) (2015).

[22] Liu, C., van der Wel, R., Rotenberg, N., Kuipers, L., Krauss, T., Di Falco, A., and Fratalocchi, A., "Triggering extreme events at the nanoscale in photonic seas," Nature Physics (2015).

[23] Totero Gongora, J. S. and Fratalocchi, A., "Harnessing Disorder at the Nanoscale," in [Encyclopedia of Nanotechnology], Bhushan, B., ed., 1-13, Springer Netherlands (2015). DOI: 10.1007/978-94-007-61780_101015-1.

[24] Andreasen, J., Cao, H., Taflove, A., Kumar, P., and Cao, C.-q., "Finite-difference time-domain simulation of thermal noise in open cavities," Phys. Rev. A 77, 023810 (Feb 2008).

[25] Lu, Y.-J., Kim, J., Chen, H.-Y., Wu, C., Dabidian, N., Sanders, C. E., Wang, C.-Y., Lu, M.-Y., Li, B.-H., Qiu, X., Chang, W.-H., Chen, L.-J., Shvets, G., Shih, C.-K., and Gwo, S., "Plasmonic Nanolaser Using Epitaxially Grown Silver Film," Science 337, 450-453 (July 2012).

[26] Ho, J., Tatebayashi, J., Sergent, S., Fong, C. F., Iwamoto, S., and Arakawa, Y., "Low-threshold near-infrared gaasalgaas coreshell nanowire plasmon laser," ACS Photonics 2(1), 165-171 (2015).

[27] Mueller, J. P. B. and Capasso, F., "Asymmetric surface plasmon polariton emission by a dipole emitter near a metal surface," Phys. Rev. B 88, 121410 (Sep 2013).

[28] Rodríguez-Fortuño, F. J., Marino, G., Ginzburg, P., O'Connor, D., Martínez, A., Wurtz, G. A., and Zayats, A. V., "Near-field interference for the unidirectional excitation of electromagnetic guided modes," Science 340(6130), 328-330 (2013). 